\title{
QCD at forward rapidity, in ultra-peripheral collisions, and multi-parton interactions
}

\section{Evgeny Kryshen for the ALICE, ATLAS, CMS and LHCb Collaborations}

National Research Center "Kurchatov Institute",

Petersburg Nuclear Physics Institute (PNPI), Gatchina, 188300, Russia

E-mail: evgeny.kryshen@cern.ch

The LHC is a unique laboratory to perform studies of diffractive processes, photon-induced reactions and multi-parton interactions and explore various aspects of non-perturbative QCD such as Pomeron structure, low- $x$ evolution of parton density functions and the dynamics of hadron collisions at non-perturbative scales. Recent results on these subjects from the ALICE, ATLAS, CMS, LHCb and TOTEM collaborations at the LHC are reviewed.

Sixth Annual Conference on Large Hadron Collider Physics (LHCP2018)

4-9 June 2018

Bologna, Italy 


\section{Introduction}

Diffractive processes, photon-induced reactions and multi-parton interactions are used to investigate various open questions of non-perturbative QCD such as Pomeron structure, low- $x$ evolution of parton density functions and the dynamics of hadron collisions at non-perturbative scales. The LHC serves as a unique laboratory to answer these questions. Recent results on diffraction, photon-induced reactions and multi-parton interactions from the ALICE, ATLAS, CMS, LHCb and TOTEM collaborations at the LHC are reviewed in the following sections.

\section{Forward physics and diffraction}

The scope of diffractive studies at the LHC ranges from conventional total and diffractive cross section measurements to various studies of hard diffractive processes. The total protonproton (pp) cross section at a center-of-mass energy $\sqrt{s}=13 \mathrm{TeV}$ has been recently measured by the TOTEM experiment with a luminosity independent method confirming the trend seen at lower energies [1]. TOTEM also managed to access very low values of the square of the transferred momentum ( $t$ ) down to $|t|=8 \cdot 10^{-4} \mathrm{GeV}^{2}$, dominated by the Coulomb interaction [2]. Surprisingly the $\rho$ parameter, the real to imaginary ratio of the nuclear elastic scattering amplitude at $t=0$ extracted from the interference of Coulomb and nuclear interactions, appeared to be much lower than expected from previous measurements, see Fig. 1, left. Traditional Pomeron-exchange models failed to describe the behaviour of the total cross section and the $\rho$ parameter simultaneously with one set of parameters suggesting an unexpectedly significant contribution of the Odderon exchange (the odd-under-crossing counterpart of the Pomeron).

The value of the total cross section and the ratio of elastic and inelastic event counts allowed TOTEM to measure the inelastic cross section at $\sqrt{s}=13 \mathrm{TeV}$ with unprecedented $2 \%$ precision: $\sigma_{\mathrm{INEL}}=79.5 \pm 1.8 \mathrm{mb}$. LHCb and ATLAS also extracted the inelastic cross section with a conventional method based on the measurement of the visible cross section in minimum-bias events: $\sigma_{\mathrm{INEL}}=75.4 \pm 3.0$ (exp.) \pm 4.5 (extr.) $\mathrm{mb}[3]$ and $\sigma_{\mathrm{INEL}}=78.1 \pm 0.6$ (exp.) \pm 1.3 (lumi.) \pm 2.6 (extr.) $\mathrm{mb}$ [4], respectively. Results are consistent with the TOTEM value with the dominant source of uncertainty coming from the extrapolation of the visible cross sections to low diffractive masses $\left(M_{X}<13 \mathrm{GeV}\right.$ in ATLAS). CMS managed to measure fiducial cross sections at much lower diffractive masses down to $M_{X} \sim 4.1 \mathrm{GeV}$ [5]. The measured cross sections are smaller than those predicted by the majority of models thus setting important constraints on Pomeron dynamics and low-mass diffractive contributions.

Pomeron physics can be further studied with central exclusive events dominated by pomeronpomeron interactions. CMS has recently published the first measurement of central exclusive pionpair production at the LHC [6]. ALICE has also collected a large sample of central exclusive events, allowing one to extract poorly known pomeron-meson effective couplings, perform partial wave analyses and precision spectroscopy of hadron resonances, and search for glueballs and other exotic states.

Studies of diffractive dijet production in events with large rapidity gaps provide additional input on the pomeron dynamics. ATLAS measured dijet cross sections as a function of rapidity gap and as a function of $\xi$, the proton's longitudinal momentum carried by the Pomeron [7], see 

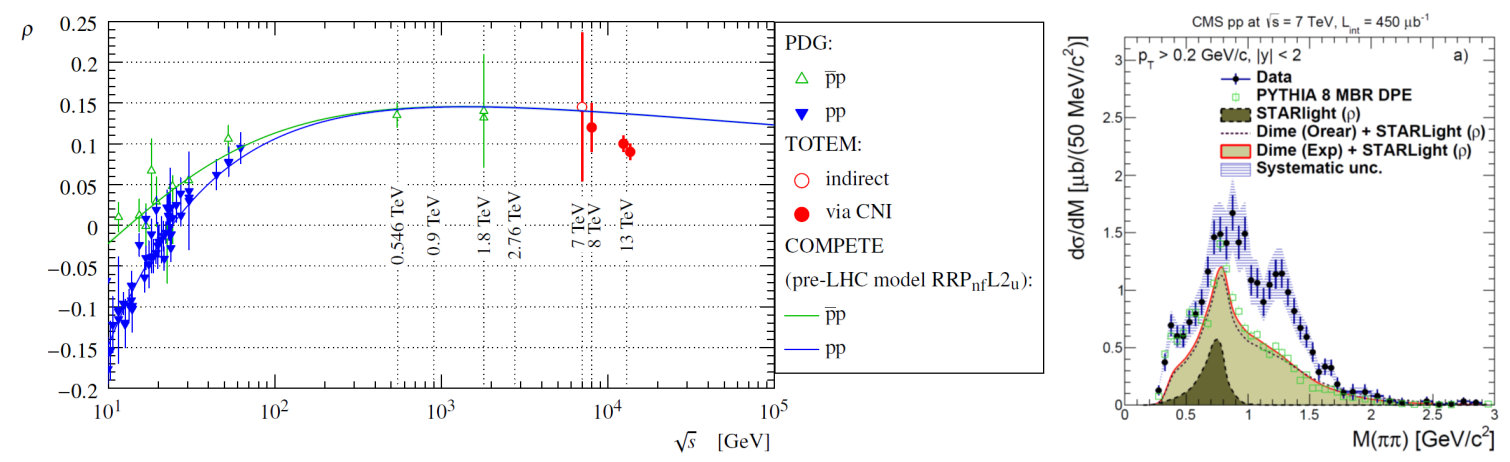

Figure 1: Left: $\rho$ parameter measured by TOTEM [2]. Right: differential cross section of central exclusive pion-pair production measured by CMS [6].

Fig. 2, left. The measured cross section appears to be a factor of 6 lower than theory predictions based on diffractive PDFs extracted from HERA data, the discrepancy being explained by the high probability of multiple soft interactions between protons spoiling rapidity gaps [8].

Hard pomeron dynamics and BFKL effects can also be investigated with jet-gap-jet events characterized by color-singlet (hard pomeron) exchange between jets. CMS measured the fraction of events with a rapidity gap in $|\eta|<1$ as a function of the jet transverse momentum [9], see Fig. 2, right. The fraction of color-singlet exchange events is lower compared to Tevatron energies in qualitative agreement with models; however, quantitatively this fraction appears to be much smaller than BFKL predictions indicating the importance of soft rescattering effects.

\section{Photon-induced reactions}

Photon-induced reactions become dominant in ultra-peripheral collisions with impact parameters larger than the sum of hadron radii [10]. In the Weizsäcker-Williams approximation, strong electromagnetic fields of relativistic hadrons are equivalent to the flux of quasi-real photons which is proportional to the squared hadron charge $\mathrm{Z}$. This strong photon flux results in large cross sec-
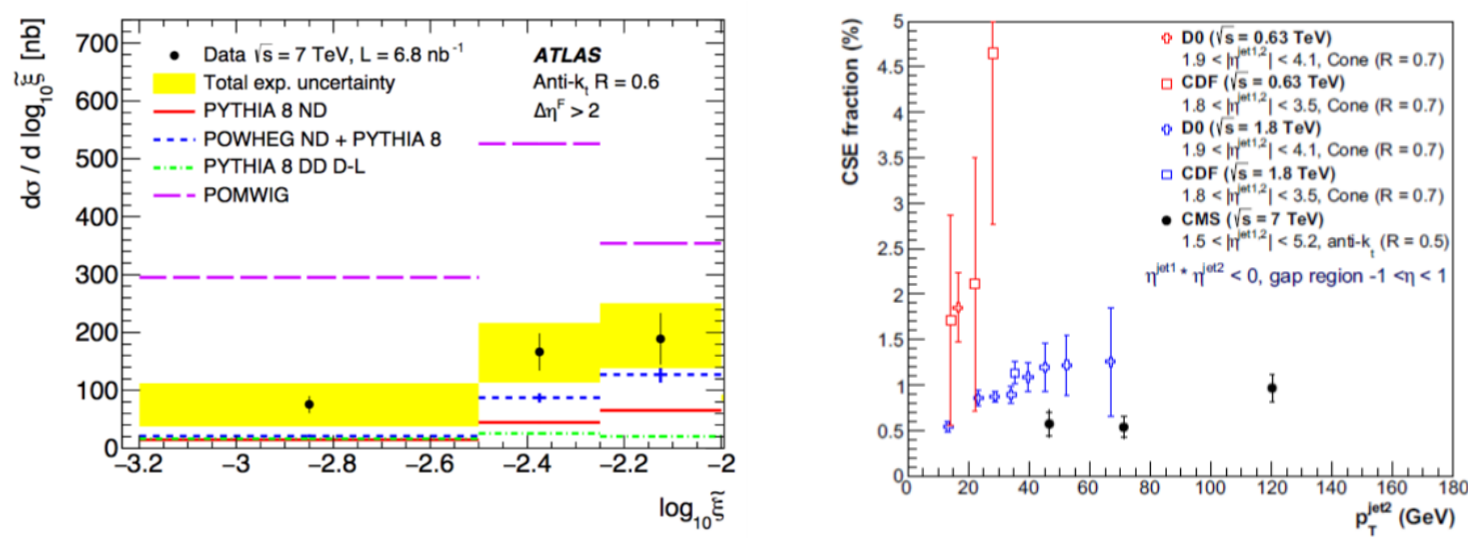

Figure 2: Left: the differential cross section of diffractive dijet production measured by ATLAS [7]. Right: the fraction of color-singlet-exchange events measured by the CMS, CDF and D0 experiments [9]. 
tions for $\gamma \mathrm{Pb}$ and $\gamma \mathrm{p}$ and $\gamma \gamma$ interactions at center-of-mass energies much higher than achieved before.

Dilepton photoproduction is one of the simplest examples of photon-induced reactions. It can be factorized into the photon flux and the $\gamma \gamma \rightarrow l l$ cross section precisely calculated in LO QED. ATLAS has measured the spectrum of dimuons in pp collisions at $\sqrt{s}=13 \mathrm{TeV}$ and found $10-20 \%$ lower values than expected in the equivalent photon approximation [11], see Fig. 3, right. This difference is attributed to extra hadronic interactions between colliding protons, absorptive corrections being expected to increase with dimuon mass. Experimental uncertainties can be potentially improved with proton tagging, see the first CMS+TOTEM measurements at dimuon masses above $100 \mathrm{GeV}$ with protons tagged in TOTEM Roman Pots [12].

Photon-photon interactions become much more abundant in $\mathrm{Pb}-\mathrm{Pb}$ collisions since their cross section scales as $Z^{4}$. Dielectron and dimuon cross sections measured by ALICE, ATLAS and CMS agree with leading order QED calculations in a wide range of invariant masses up to 100 GeV [13, 14, 15], see Fig. 3, right, for example.

Strong electromagnetic fields of incoming nuclei at the LHC allowed ATLAS and CMS to perform unique light-by-light scattering measurements potentially sensitive to anomalous gauge couplings and contributions from BSM particles. The measured diphoton spectra have a significance of $\sim 4 \sigma$ both in ATLAS and CMS indicating good agreement with Standard Model predictions [13, 16], see Fig. 4.

Heavy vector meson photoproduction measurements in ultra-peripheral collisions at the LHC allow one to probe poorly known gluon distributions at low $x$ and search for gluon saturation effects because, in $\mathrm{pQCD}$ at $\mathrm{LO}$, the coherent photoproduction cross section is proportional to the square of the gluon density in the target hadron. The acceptance coverage of the LHC experiments corresponds to a Bjorken- $x$ range from $\sim 10^{-2}$ to $\sim 10^{-5}$, while the heavy quark mass serves as a hard scale justifying perturbative calculations.

Exclusive $J / \psi$ photoproduction off protons has been measured in pp collisions by LHCb and in $\mathrm{p}-\mathrm{Pb}$ collisions by ALICE extending the explored range of $\gamma p$ center-of-mass energies to the TeV scale [17, 18], see Fig. 5, left. The energy dependence of the photoproduction cross section is compatible with a power law showing no significant change in the gluon density behavior down to
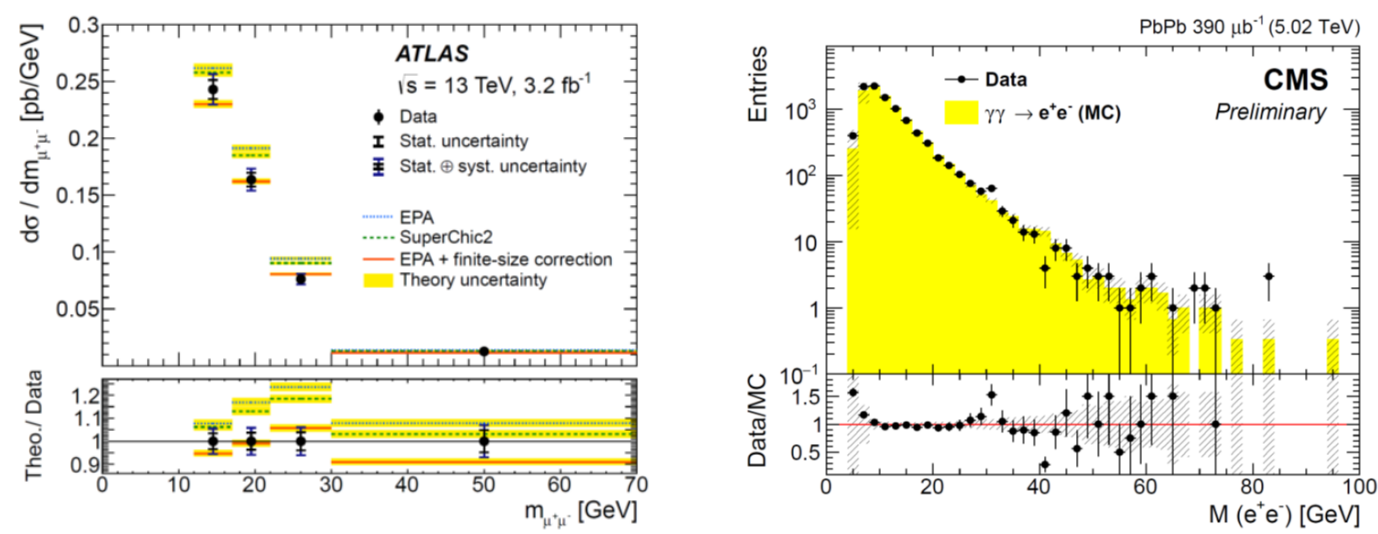

Figure 3: Left: dimuon photoproduction cross section in pp collisions at $\sqrt{s}=13 \mathrm{TeV}$ measured by ATLAS [11]. Right: dielectron photoproduction cross section in Pb-Pb collisions measured by CMS [13]. 

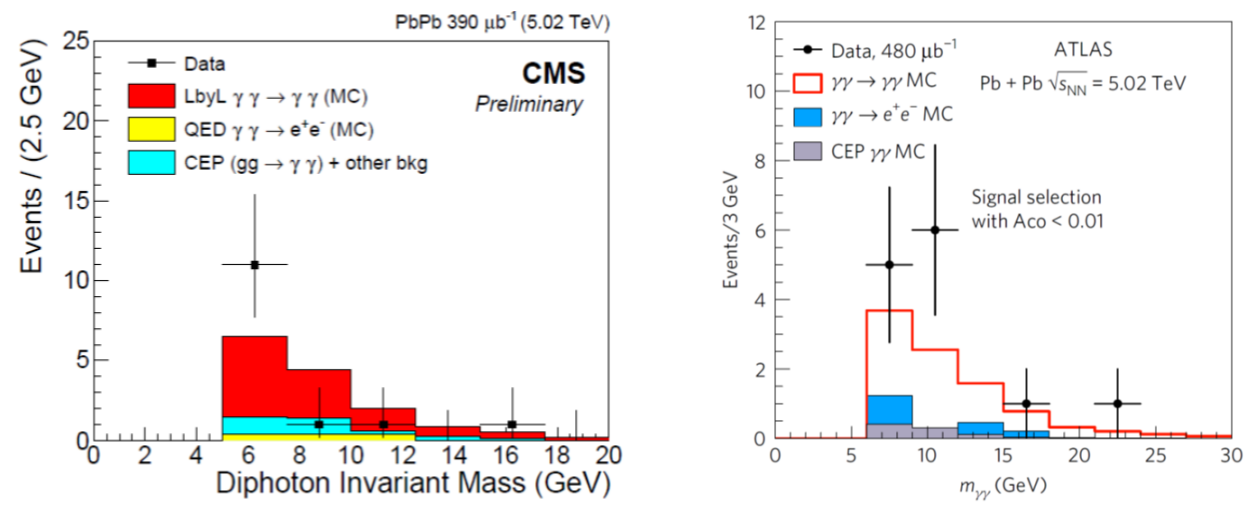

Figure 4: Light-by-light scattering cross sections as a function of diphoton invariant mass measured by CMS (left) and ATLAS (right) [13, 16].

$x \sim 10^{-5}$ at $Q^{2} \sim \frac{1}{4} m_{J / \psi}^{2}$.

Measurements of $\psi(2 S)$ or $\Upsilon$ photoproduction would allow one to probe gluon densities at larger scales. $\psi(2 S)$ and $\Upsilon(1 S)$ photoproduction cross sections measured by LHCb in pp collisions [17, 19] and $\Upsilon(1 S)$ photoproduction cross sections measured by CMS in p-Pb collisions [20] are compatible with a power-law growth of gluon densities, however the comparison of $\Upsilon$ measurements with theory calculations indicate the importance of NLO effects, see Fig. 5, right.

Quarkonium photoproduction off a $\mathrm{Pb}$ target serves as a tool to probe gluon distributions and to study poorly known gluon shadowing effects in Pb. ALICE and CMS results of coherent $J / \psi$ photoproduction in Pb-Pb UPC from Run 1 [15, 21, 22] appeared to be in good agreement with models based on the EPS09 parameterization of gluon shadowing and were interpreted as the first direct observation of strong gluon shadowing effects at moderate $x$ values from $10^{-3}$ to $10^{-2}$ [23]. Preliminary ALICE and LHCb results on $J / \psi$ photoproduction at forward rapidity in $\mathrm{Pb}-\mathrm{Pb}$ UPC from Run 2 provide much better precision compared to previous Run 1 measurements, see Fig. 6.

Another tool to probe the partonic structure of nuclei is to explore photonuclear dijet production in $\mathrm{Pb}-\mathrm{Pb}$ UPC and select events with intact ions by vetoing neutron activity in one of the zero-degree calorimeters. Preliminary results from ATLAS illustrate the high potential of this
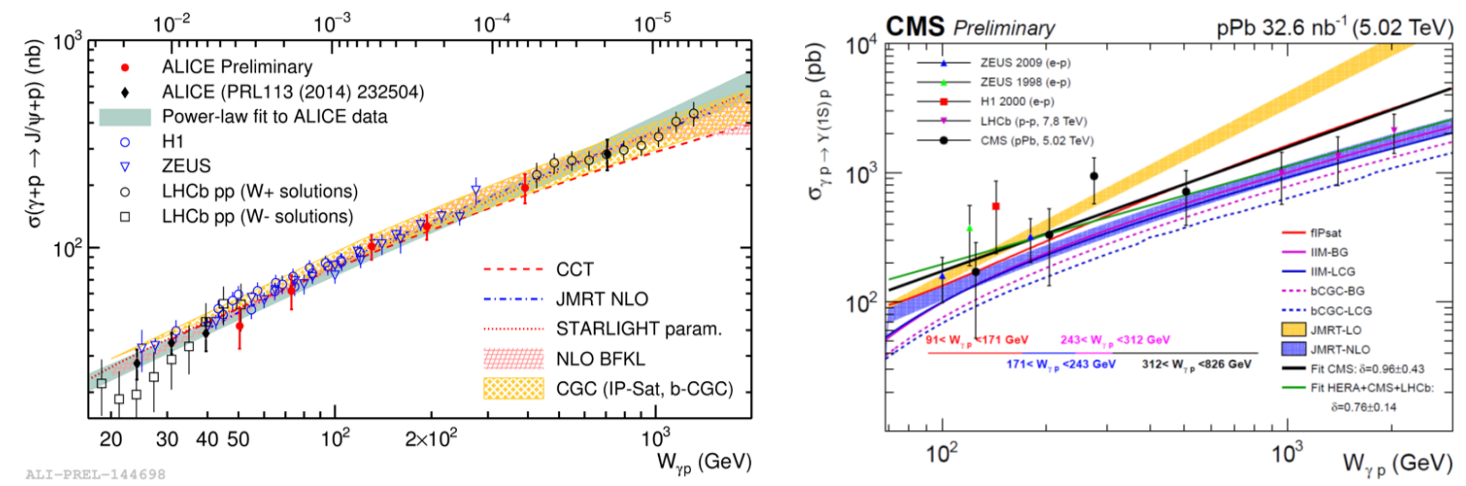

Figure 5: $J / \psi$ (left) and $\Upsilon$ (right) photoproduction cross section off protons measured by the ALICE, ATLAS and LHCb experiments. 

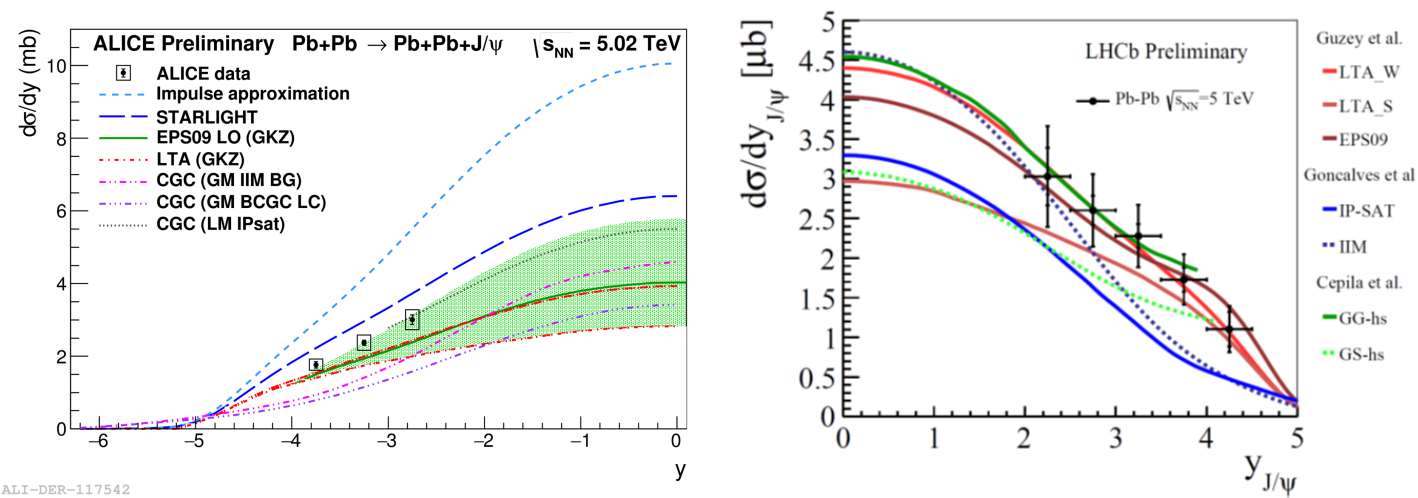

Figure 6: Coherent $J / \psi$ photoproduction cross section in Pb-Pb UPC measured by the ALICE (left) and $\mathrm{LHCb}$ (right) experiments.

technique [24].

Although photon-induced reactions are typically measured in UPCs, they have also been observed in hadronic collisions of heavy ions. A strong excess in the yield of $J / \psi$ at very low transverse momenta in peripheral $\mathrm{Pb}-\mathrm{Pb}$ collisions measured by ALICE [25] is commonly interpreted as a signal of coherent $J / \psi$ photoproduction off heavy ion remnants. Dimuon photoproduction has been also studied in peripheral and central $\mathrm{Pb}-\mathrm{Pb}$ collisions by ATLAS [26]. The dimuon acoplanarity distribution was found to systematically broaden towards more central collisions. This observation can probably be explained by scattering of muons off charged constituents in the hot and dense medium thus providing a new tool to probe hadronic matter produced in heavy ion collisions.

\section{Multi-parton interactions}

Multi-parton interactions (MPI) become increasingly important with growing center-of-mass energy especially for the modeling of soft particle production and for the understanding of backgrounds in measurements of various rare processes. First LHC data revealed the significance of color reconnection effects and correlations between partons [27]. Now MPIs are considered to be at the origin of collective effects in high-multiplicity pp collisions, e.g. the near-side ridge in two-particle correlations discovered by CMS [28] and the strangeness enhancement measured by ALICE [29].

There are three distinct fields of MPI studies depending on the momentum range of the scattered partons. First, one can study soft MPI effects via multi-differential measurements of particle distributions in minimum-bias and high-multiplicity collisions. Second, one can explore events with one hard parton scattering and investigate the influence of these hard scatters on average event properties and underlying events. Finally, one can select events with two hard processes, measure double parton scattering (DPS) cross sections and compare them with pQCD calculations. Recent results from the LHC Run 2 in these three domains are discussed in this section.

The ATLAS and CMS experiments have recently published a set of studies on charged particle distributions at $\sqrt{s}=13 \mathrm{TeV}$, in particular charged track density $(d N / d \eta)$ measurements, $p_{\mathrm{T}}$ and multiplicity distributions and $\left\langle p_{\mathrm{T}}\right\rangle$-multiplicity correlations [30, 31, 32]. The data is compared to various Monte Carlo generators, such as EPOS and PYTHIA, tuned to reproduce similar ob- 
servables at lower energies. The EPOS generator provides the best description for $d N / d \eta$ and $p_{\mathrm{T}}$ spectra but none of the models catches the shape of multiplicity distributions. Moreover, the $\left\langle p_{\mathrm{T}}\right\rangle$ vs-multiplicity distribution, being very sensitive to correlation effects in MPI, is not reproduced by standard PYTHIA tunes, see Fig. 7, left, thus providing new constraints for MPI models.

One can also study various observables as a function of event multiplicity, which is roughly proportional to the number of multi-parton interactions. ALICE has set up a special high-multiplicity trigger program aimed to extend the multiplicity reach up to $\sim 10\langle d N / d \eta\rangle$. In the first step, $d N / d \eta$ distributions with $|\eta|<1.5$ were studied in forward multiplicity slices and compared to EPOS and various PYTHIA tunes. Both generators provide a reasonable description of the data at large forward multiplicities but tend to underestimate the central charged particle density at moderate forward multiplicities, see Fig. 7, middle.

Electromagnetic and hadronic energy flow in the forward region $(-6.6<\eta<-5.2)$ was also studied by CMS with the CASTOR detector [33]. The total-energy spectrum is shown in Fig. 7, right, in comparison with various PYTHIA tunes. The spectrum appears to be highly sensitive to MPI dynamics, in particular to the $p_{\mathrm{T}}$ threshold (pt0Ref) setting used to control MPI in PYTHIA therefore these results can contribute to improvements in future Monte Carlo parameter tunes.

Underlying event measurements are usually performed in "transverse", "toward" and "away" regions depending on the azimuthal angle with respect to the direction of the leading charged particle. The majority of underlying-event observables study the dependences of various averaged quantities in the "transverse" region on $p_{\mathrm{T}}$ of the leading object allowing one to trace a smooth transition from minimum-bias interactions to the hard-scattering regime. For example, Fig. 8, left, illustrates the behavior of the mean scalar $p_{\mathrm{T}}$ sum of charged particles per unit solid angle as a function of the transverse momentum of the leading particle measured by ATLAS and ALICE in pp collisions at $\sqrt{s}=13 \mathrm{TeV}$. This behavior is reasonably well described by PYTHIA tuned to reproduce underlying events in lower energy data, while the EPOS generator, specialized to simulate inclusive soft QCD processes, shows up to $20 \%$ discrepancies thus casting doubt on its suitability for modelling multiple interactions despite currently providing the best description of minimum-bias data [34].
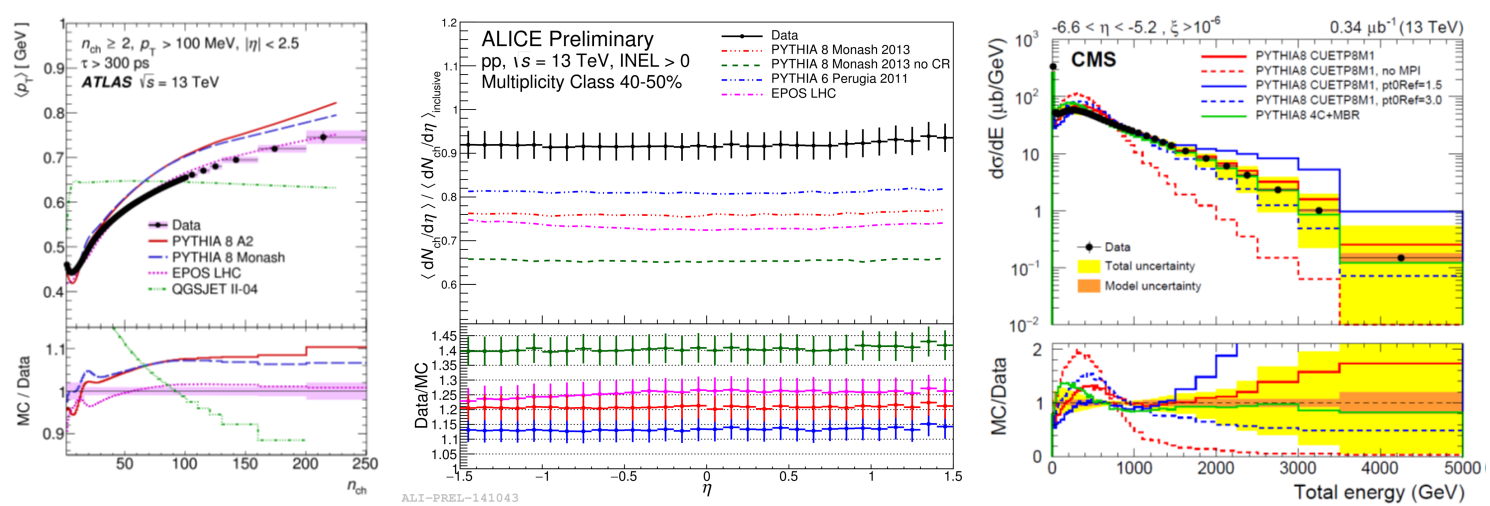

Figure 7: Left: $\left\langle p_{\mathrm{T}}\right\rangle$ distribution as a function of charged particle multiplicity measured by ATLAS in pp collisions at $\sqrt{s}=13 \mathrm{TeV}$. Middle: $d N / d \eta$ distributions in $40-50 \%$ forward multiplicity class measured by ALICE in pp collisions at $\sqrt{s}=13 \mathrm{TeV}$. Right: total energy flow at forward rapidity measured by CMS [33]. 
Double Parton Scattering (DPS) involves two hard scatterings in a single proton-proton collision and is reasonably well described in a PQCD framework allowing one to study MPI in a more controlled way. A simple model, that assumes transverse and longitudinal factorization of the two single parton interactions and ignores correlations between partons, results in a pocket formula for the DPS cross section $\sigma_{\mathrm{DPS}}^{A B}=\frac{m}{2} \sigma_{\mathrm{SPS}}^{A} \sigma_{\mathrm{SPS}}^{B} / \sigma_{\text {eff }}$ where $\sigma_{\mathrm{SPS}}^{A / B}$ are corresponding single parton scattering (SPS) cross sections, $m=1$ if $A$ and $B$ are identical and $m=2$ otherwise. The effective cross section $\sigma_{\text {eff }}$ is related to the geometrical distribution of scattered partons and is supposed to be independent of the processes $A / B$ and collision energy in this simple model. DPS has been studied at the Tevatron and LHC in various processes like 4 jets, $\gamma+3$ jets, $W+2$ jets, $J / \psi+D, D D$ etc. The measured $\sigma_{\text {eff }}$ values lie around $15-20 \mathrm{mb}$ with no particular process or energy dependence observed within uncertainties.

Same-sign WW production is one of the most promising processes to study DPS since the corresponding SPS process with a same-sign WW is accompanied by two additional high-energy partons in the final state and is easily suppressed by introducing an upper limit on the number of jets. Fig. 8, right, shows first CMS results on $\sigma_{\text {eff }}$ measurements in same-sign WW events in comparison with $\sigma_{\text {eff }}$ extracted in previous DPS measurements $[35,36]$. Theoretically, this process will eventually permit to study the validity of the factorization approach and nontrivial longitudinal correlations among the partons.
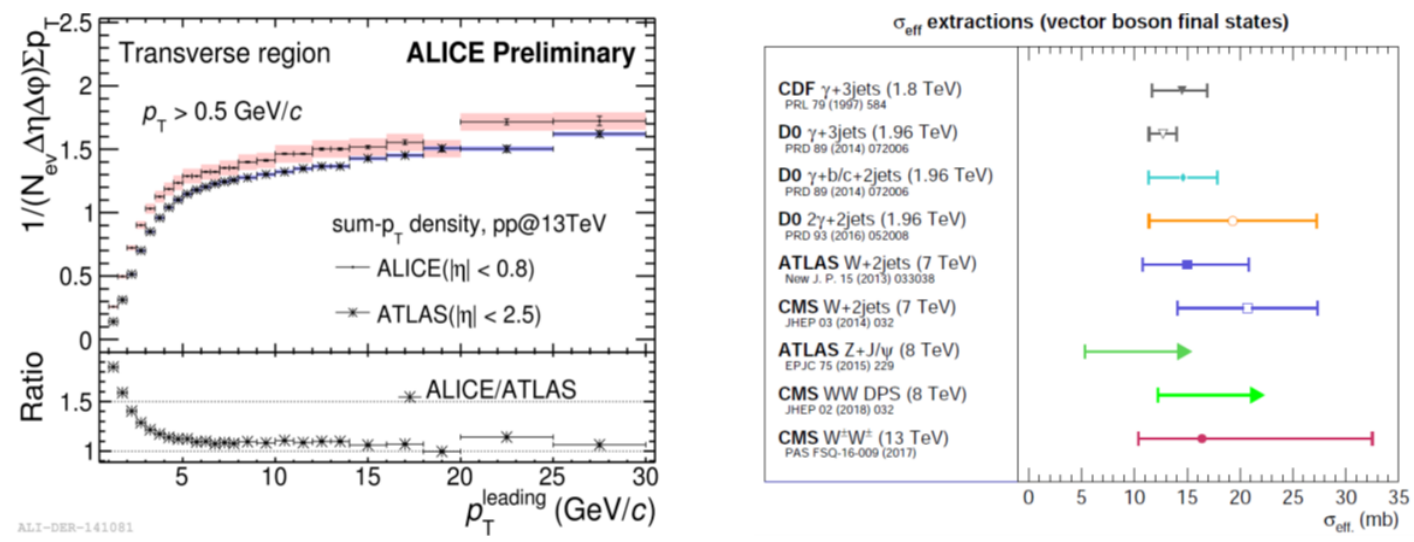

Figure 8: Left: mean scalar $p_{\mathrm{T}}$ sum of charged particles per unit solid angle as a function of the leading $p_{\mathrm{T}}$ measured by ALICE and ATLAS. Right: $\sigma_{\text {eff }}$ values extracted in various DPS studies [35, 36].

\section{Conclusions}

Over the past few years, many exciting results on diffractive and photon-induced processes have appeared. TOTEM has uncovered possible evidence of the Odderon that until now had only been theorized to exist. Light-by-light scattering has been finally observed in UPC providing new tools to set limits on BSM physics. Vector meson photoproduction has shown to be particularly useful as a probe of nuclear structure. The intense analysis of the LHC Run 2 data has brought a substantial confirmation of our understanding and increased comprehension of the role played by MPI in hadron collisions. The upcoming high-luminosity LHC era will bring much more precise measurements and hopefully new exciting discoveries in these fields. 


\section{References}

[1] TOTEM collaboration, arXiv:1712.06153.

[2] TOTEM collaboration, CERN-EP-2017-335 (2017).

[3] LHCb collaboration, JHEP 06 (2018) 100 [1803.10974].

[4] ATLAS collaboration, Phys. Rev. Lett. 117 (2016) 182002 [160 6.02625 ].

[5] CMS collaboration, JHEP 07 (2018) 161 [1802 . 02613].

[6] CMS collaboration, arXiv:1706.08310.

[7] ATLAS collaboration, Phys. Lett. B754 (2016) 214 [1511. 00502 ].

[8] V. A. Khoze, A. D. Martin and M. G. Ryskin, J. Phys. G45 (2018) 053002 [1 710.11505 ].

[9] CMS collaboration, Eur. Phys. J. C78 (2018) 242 [1710. 02586 ].

[10] J. G. Contreras and J. D. Tapia Takaki, Int. J. Mod. Phys. A30 (2015) 1542012.

[11] ATLAS collaboration, Phys. Lett. B777 (2018) 303 [1708. 04053 ].

[12] CMS and TOTEM collaborations, JHEP 1807 (2018) 153 [1803. 04496 ].

[13] CMS collaboration, CMS-PAS-FSQ-16-012 (2018) .

[14] ATLAS collaboration, ATLAS-CONF-2016-025 (2016) .

[15] ALICE collaboration, Eur. Phys. J. C73 (2013) 2617 [1305 . 1467].

[16] ATLAS collaboration, Nature Phys. 13 (2017) 852 [1702.01625].

[17] LHCb collaboration, LHCb-CONF-2016-007 (2016) .

[18] ALICE collaboration, Phys. Rev. Lett. 113 (2014) 232504 [1406. 7819 ].

[19] LHCb collaboration, JHEP 09 (2015) 084 [1505 . 08139].

[20] CMS collaboration, CMS-PAS-FSQ-13-009 (2016) .

[21] ALICE collaboration, Phys. Lett. B718 (2013) 1273 [1209.3715].

[22] CMS collaboration, Phys. Lett. B772 (2017) 489 [1605.06966].

[23] V. Guzey, E. Kryshen, M. Strikman and M. Zhalov, Phys. Lett. B726 (2013) 290 [1305. 1724 ].

[24] ATLAS collaboration, ATLAS-CONF-2017-011 (2017).

[25] ALICE collaboration, Phys. Rev. Lett. 116 (2016) 222301 [1509. 08802].

[26] ATLAS collaboration, arXiv:1806.08708.

[27] P. Bartalini, Mod. Phys. Lett. A28 (2013) 1330010.

[28] CMS collaboration, JHEP 09 (2010) 091 [1009.4122].

[29] ALICE collaboration, Nature Phys. 13 (2017) 535 [1606 . 07424].

[30] CMS collaboration, CMS-PAS-FSQ-15-008 (2016) .

[31] ATLAS collaboration, Eur. Phys. J. C76 (2016) 502 [1606. 01133 ].

[32] ATLAS collaboration, Phys. Lett. B758 (2016) 67 [1602.01633].

[33] CMS collaboration, JHEP 08 (2017) 046 [1701. 08695 ].

[34] ATLAS collaboration, JHEP 03 (2017) 157 [1 701 . 05390 ].

[35] CMS collaboration, JHEP 02 (2018) 032 [1 712 . 02280].

[36] CMS collaboration, CMS-PAS-FSQ-16-009 (2017) . 